\title{
O QUE PODEMOS APRENDER COM A INTERFACE DO FACEBOOK?
}

\author{
Luís Henrique Lindner \\ Mestrando EGC/UFSC \\ luishenrique87@gmail.com \\ Vânia Ribas Ulbricht, Dra. \\ Professora do PPGEGC/UFSC \\ vrulbricht@gmail.com
}

\begin{abstract}
Resumo: A interface gráfica exerce grande influência na experiência dos usuários. Recebe ainda mais atenção quando se trata da interface de um site acessado por 1,2 bilhão de usuários, o Facebook. Este artigo apresenta uma análise da interface da plataforma com objetivo de identificar suas características, elementos visuais e os fatores que possam justificar o layout atual. Para tal, é realizada uma pesquisa bibliográfica na própria rede social, em blogs e portais de notícias. A partir da análise, observa-se que a interface é uma mídia em constante evolução, diretamente influenciada pela tecnologia. Além disso, destaca-se elementos que interferem no engajamento dos usuários, como uso de imagens e os campos para inserção de conteúdo.
\end{abstract}

Palavras-chave: Interface, Redes Sociais, Facebook

Abstract: The graphical interface has great influence on user experience. Also gets attention when it comes to the interface a website accessed by 1.2 billion users, the Facebook This article presents an analysis of the platform in order to identify its characteristics, visual elements and factors that may justify the current layout interface. To this end, a literature search is performed on the social network itself, blogs and news portals. From the analysis, it is observed that the interface is a media constantly evolving, directly influenced by technology. Furthermore, we highlight elements that interfere with the engagement of users, such as use of images and fields for inserting content.

Key words: Interface, Social Media, Facebook 


\section{INTRODUÇÃO}

Segundo dados do NetView, uma publicação do IBOPE Media (IBOPE, 2013), $86 \%$ dos internautas ativos no Brasil são usuários de redes sociais, o que representa um crescimento de $15 \%$ em relação à 2012. Entre as redes sociais, destaca-se o Facebook (FOLHA, 2012), uma plataforma massiva com conteúdo gerado pelos usuários, por páginas de empresas e grupos.

O Facebook é recente, surgiu em 2004, mas já está estabelecido como meio de comunicação na sociedade, presente em diversos âmbitos da vida, seja pessoal, acadêmica ou profissional. Seu caráter generalista permite conteúdos sobre diversos assuntos, incluindo relatos e atualizações de status.

Segundo o Mapa Mundial de Redes Sociais (COSENZA, 2013), o Facebook é a rede social preferida em 127 países de 137 pesquisados, totalizando cerca de 1,2 bilhão de usuários. Uma rede social com esta dimensão pode ser analisada sob diversos aspectos, entre eles a interface, crucial para navegação dos usuários. Nesse sentido, este artigo busca identificar o que podemos aprender com a interface do Facebook. Para tal, realiza-se uma pesquisa descritiva com base na última atualização da interface, indicando as alterações realizadas e opiniões de especialistas em resenhas disponíveis online.

\section{INTERFACE}

O conceito de interface ter origem com a era da informática e dos sistemas (BONSIEPE, 1997), em que o ser humano precisava de um meio para "conversar" e interagir com a máquina.

Johnson (2001, p. 17) afirma que

A interface atua como uma espécie de tradutor, mediando entre as duas partes, tornando uma sensível para a outra. Em outras palavras, a relação governada pela interface é uma relação semântica, caracterizada por significado e expressão, não por força física.

O autor ressalta a importância da metáfora na interface tratando essencialmente do meio digital, no qual, segundo ele, o ser humano não interage com a informação real - que está na forma de zeros e uns - mas com uma representação gráfica em forma de metáforas. Esta ideia, que popularizou o conceito de interface gráfica do usuário - GUI, gerou uma atividade de design de interfaces voltada para representação das informações e dos sistemas eletrônicos. Surgem diversas convenções, como o uso de janelas para diferentes aplicações e textos em azul e sublinhados para hiperlinks, voltadas para facilitar a navegação dos usuários da execução das tarefas.

A ISO 9241-11 de 1998 afirma que "usabilidade é a capacidade de um produto ser usado por usuários específicos para atingir objetivos específicos com eficácia, eficiência e satisfação em contexto de uso específico".

Nesse sentido, é inegável a importância da tarefa na concepção de um artefato funcional. Contudo, apesar dos avanços no campo da usabilidade, muito tem se discutido acerca da satisfação do usuário. Para alguns autores o item satisfação, presente no texto da ISO 9241-11, estaria relacionada simplesmente à finalização da 
tarefa. Para outros, a satisfação vai além. Por que alguns sites com possuem baixa legibilidade e informações desorganizadas continuam preferidos por alguns usuários? Norman (2008) aponta alguns caminhos ao tratar do Design Emocional.

Segundo Norman (2008), as pessoas podem se relacionar com artefatos em três níveis: visceral, comportamental e reflexivo. O primeiro relacionado à impressão inicial estética, o segundo, voltado para o uso e o último focado no valor simbólico da relação.

A partir da visão de Norman (2008), podemos resgatar o ponto de vista de Bonsiepe (1997), que propõe um diagrama ontológico do Design composto de usuário, tarefa e artefato. O autor afirma que a interface transforma sinais em informação interpretável, revelando o potencial de artefatos materiais e comunicativos. Mas além disso, destaca que a interface é um espaço entre estes três campos e não o objeto/artefato em si, onde o usuário pode "mover-se no espaço informacional de acordo com seus interesses" (BONSIEPE, 1997, p. 59).

\section{REDES SOCIAIS}

Segundo dados da Pew Research (DUGGAN; SMITH, 2013), as redes sociais mais utilizadas em 2013 entre os adultos norte-americanos foram, na sequência, Facebook, Linkedin, Pinterest, Twitter e Instagram, conforme apresentado na Figura 1.

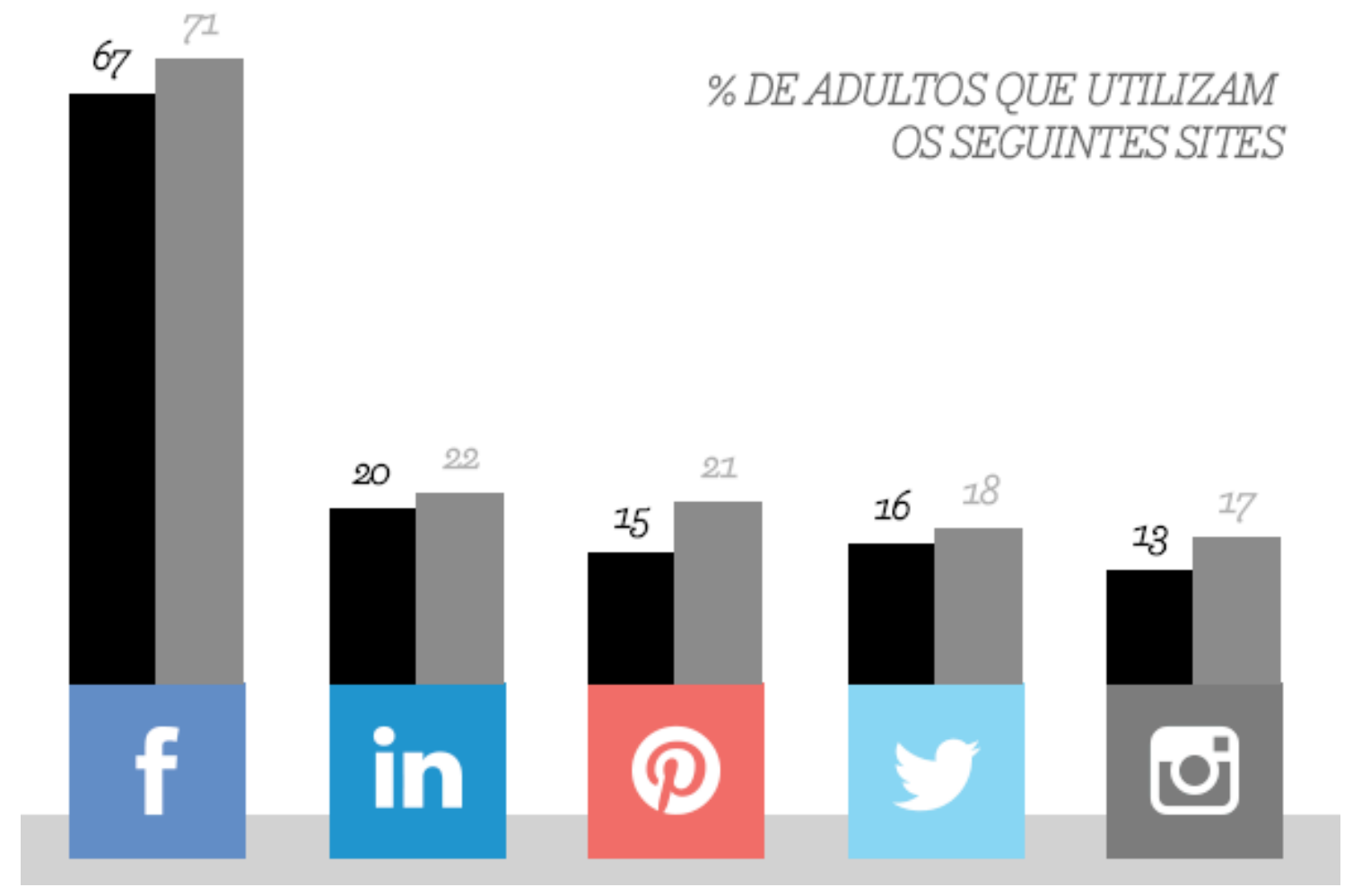

Figura 1 - Sites de redes sociais mais utilizados nos EUA. Dados de 2012 (em preto) e 2013 (em cinza) Fonte: DUGGAN; SMITH, 2013 apud LEOCADIO, 2014.

O Facebook, com uma vantagem significativa em relação as demais, foi fundado em 2004 e atingiu 1 bilhão de usuários em 2012 (FOLHA, 2012). Sua proposta é 
possibilitar na internet as relações sociais que já acontecem fora dela. Seu caráter generalista e massivo é um dos motivos pelo qual faz sucesso entre os usuários.

O Linkedin, por sua vez, é considerada uma rede para profissionais. Seu propósito é que os usuários possam estabelecer uma identidade profissional online, por meio do preenchimento dos seus dados e experiências, e, com isso, estabalecer conexões com outros profissionais e empresas. Nesse sentido, a rede oferece também oportunidades de emprego anunciadas por empresas, grupos de discussão e feeds de notícias. Tudo com objetivo de gerar insights para os profissionais e melhores opções de recrutamento para as empresas (LINKEDIN, 2014).

O Pinterest apresenta uma estrutura mais simples, comparado ao Linkedin e ao Facebook. Seu propósito, de acordo com próprio site (PINTEREST, 2014), é ser uma ferramenta para coletar e organizar coisas que inspiram as pessoas. Nesse sentido, a plataforma possibilita a criação de boards - painéis - nas quais os usuários podem incluir pins - marcadores - em forma de imagens. Os painéis auxiliam na organização dos pins e podem ser seguidos por outros usuários. Assim, além de contar com a própria coletânea de referências, o usuário pode seguir outros membro e seus painéis, a fim de enriquecer as suas fontes, comentar e trocar ideias.

O Twitter, que já foi mais popular no Brasil segundo O Globo (2012), também apresenta uma estrutura simples e direta. Segundo consta na própria rede social (TWITTER, 2014), sua proposta é ajudar a criar e compartilhar ideias e informações instantaneamente, sem barreiras, facilitando a conexão entre as pessoas, a expressão individual e a descoberta do que está acontecendo no momento. Nesse sentido, usuários podem criar um breve perfil e publicar textos de até 140 caracteres, acompanhados ou não de imagens e links. Ao seguir outros usuários, suas publicações aparecem na sua página inicial, facilitando um rápido acesso à informação.

Por fim, o Instagram, plataforma criada essencialmente para uso em smartphones, é baseada no compartilhamento de imagens. Segundo a rede (INSTAGRAM, 2014), seu objetivo é permitir que usuários compartilhem suas vidas por meio de uma série de fotos e vídeos, possibilitando o uso de filtros com diferentes efeitos, resgatando as imagens das antigas câmeras instantâneas. Usuários podem seguir outros, curtir e comentar suas fotos, além de marcar amigos sobre as imagens.

Devido ao grande número de usuários do Facebook (DUGGAN; SMITH, 2013) e pelas recentes alterações na sua interface web (FACEBOOK, 2014), optou-se por analisar os elementos visuais desta rede, com base em uma pesquisa bibliográfica, comparando opiniões de diferentes autores e publicações. A pesquisa bibliográfica, segundo Gil (2008), utiliza fontes de dados que já foram processados e analisados por algum autor, como artigos científicos, notícias ou publicações na internet. Assim, além das informações disponíveis no site institucional do Facebook, é realizada uma busca em blogs e portais de notícias para comparar opiniões e compreender suas características.

\section{INTERFACE DO FACEBOOK}

Desde sua criação em 2004, o Facebook promoveu diversas alterações em sua interface, sendo a mais recente em março de 2014. Contudo, antes de apresentar esta, 
que agora está presente nos computadores de grande parte do mundo, o Facebook havia anunciado uma outra interface, totalmente reformulada, em março de 2013 (FACEBOOK, 2013).

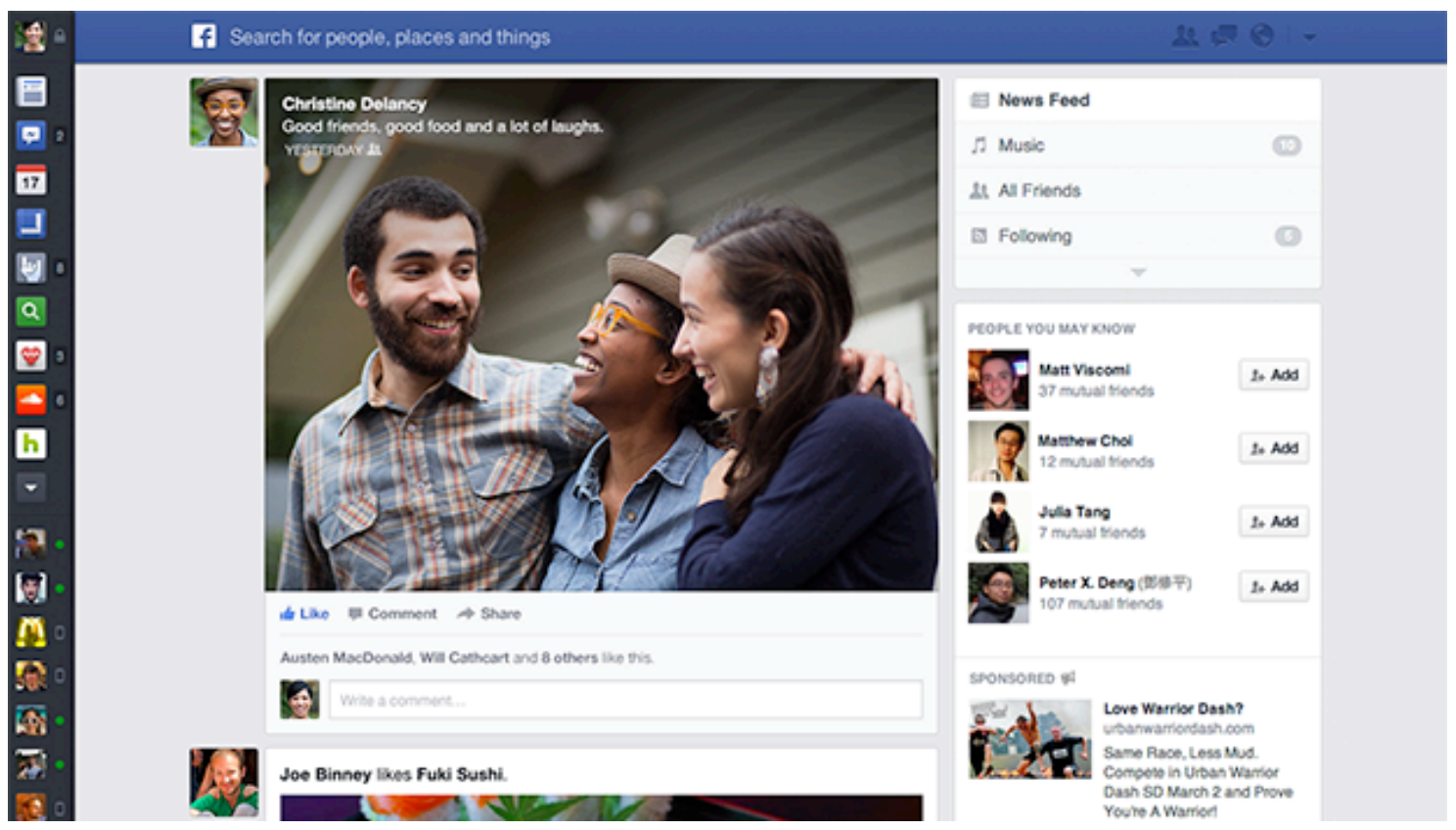

Figura 2 - Proposta de interface anunciada pelo Facebook em Março de 2013.

Fonte: FACEBOOK, 2013.

No layout anunciado em 2013 (Figura 2), o Facebook sugere uma remodelação na estrutura com objetivo de reduzir a massa de informação e ampliar o foco nas histórias das pessoas e páginas que o usuário segue. Para isso, destacou-se a área destinada aos posts, ampliando o espaço ocupado por imagens, mapas e vídeos. Current version of News Feed New version of News Feed

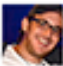

Chris Struhar likes Fuki Sush

Q Fuki Sushi

St whe page

What the Chef is preparing: Yuzu Miso Nutta Tender raw for onion slightly seasoned with a nuzu milyo aauce, Enjor

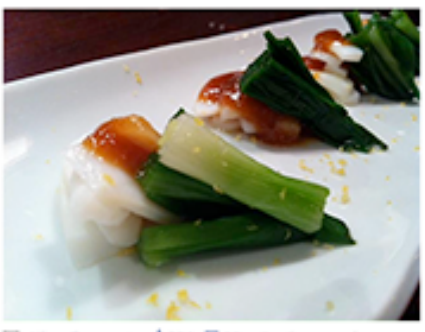

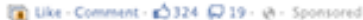

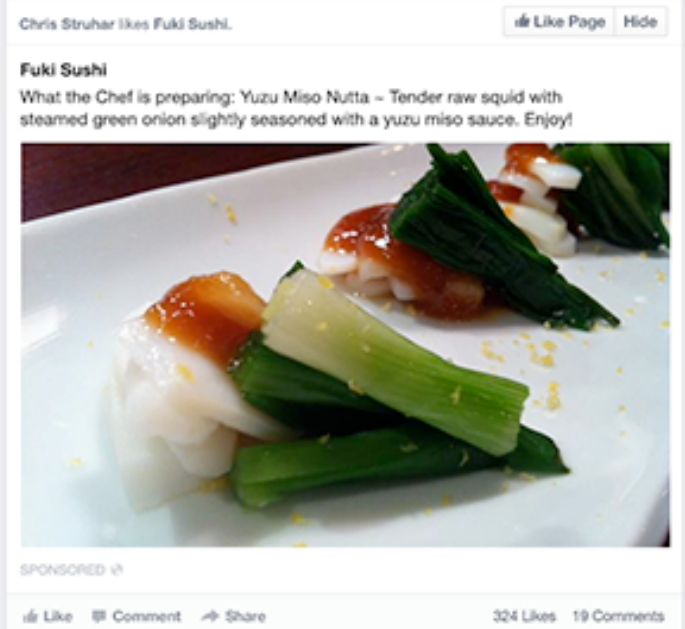

Figura 3 - Comparativo entre o layout dos posts.

Fonte: FACEBOOK, 2013. 
Observa-se na Figura 3 que a fotografia ocupa um espaço maior na tela, ficando em primeiro plano. Segundo Zuckerberg (apud CONSTINE, 2013), a proposta do Facebook é se tornar um jornal particular e personalizado, e para isso, deve oferecer conteúdo de qualidade, ser visual, rico e engajador.

Ele [o Facebook] deve apresentar mais do que apenas texto. [...] Agora que todos nós temos câmeras em nossos bolsos, feeds de notícias tornaram-se essencialmente formados por conteúdo visual. Cerca de $50 \%$ do conteúdo no feed de atividades é visual. [...] $\mathrm{A}$ forma como compatilhamos está mudando (ZUCKERBERG apud CONSTINE, 2013, online).

Esta proposta também tomou como base as interfaces da rede para dispositivos móveis, onde menus e informações secundárias ficam ocultas (FACEBOOK, 2013). Na Figura 2 é possível observar esta característica no menu à esquerda. Os rótulos foram suprimidos e os ícones ampliados.

Essa interface apresentada em 2013, no entanto, não foi implementada, segundo Facebook (2014), por causa dos relatos de usuários que participaram dos testes iniciais. De acordo com a plataforma, em geral, as pessoas gostaram das imagens grandes, mas apontaram que a navegação ficou mais difícil. Assim, o que observamos na interface lançada em 2014 é uma adaptação da proposta de 2013.

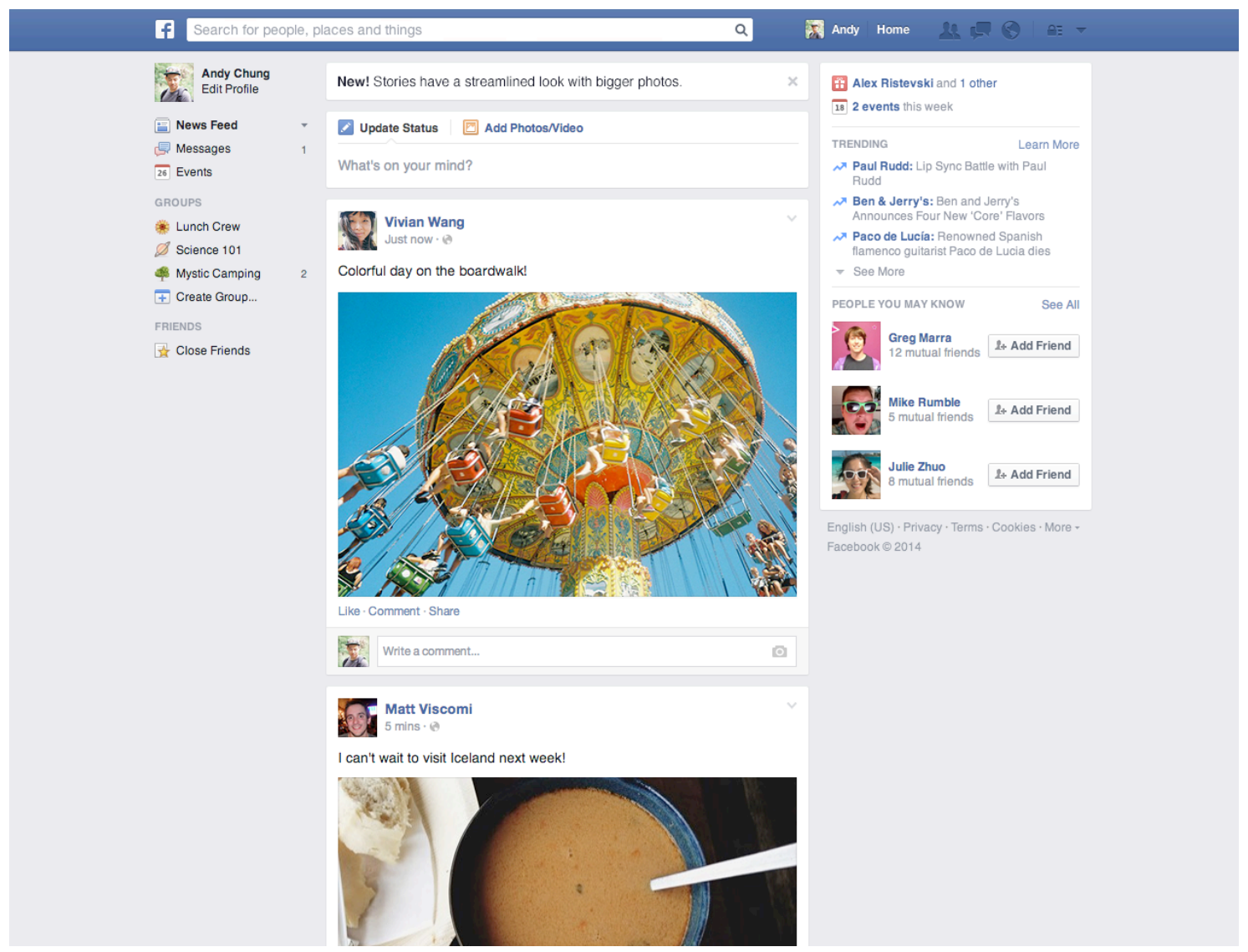

Figura 4 - Interface lançada pelo Facebook em março de 2014.

Fonte: Facebook, 2014. 
Observa-se nesta interface que as imagens seguem a proposta de ampliação, mas numa proporção um pouco menor que a sugestão anterior. Lunden (2014) afirma que parte das alterações propostas em 2013 não foram implantadas em 2014, pois ainda há um grande número de usuários que utiliza monitores com baixa resolução ou mouses sem o scroll - função de rolagem, o que teria dificultado a navegação.

As novidades na versão de 2014 se tornam mais evidentes quando a interface é comparada ao layout em uso antes da sua implementação. Segundo a FOLHA (2014), as principais alterações são a cor de fundo - que passa a ser cinza claro, e as imagens maiores. Brownlee (2014) faz uma análise mais detalhada e aponta que a nova cor de fundo destaca os posts, que continuaram em fundo branco, deixando-os em primeiro plano. Além disso, as publicações foram separadas em "cartas" - card layout, o que também facilita a percepção de início e fim de cada história (LUNDEN, 2014). Para Adams (2014), a estrutura de cartas é o futuro da web, pois elas facilitam a comunicação de histórias curtas.

A nova interface apresenta ainda outras alterações. Brownlee (2014) aponta que menu lateral esquerdo agora apresenta menos informações, mas ainda mantém os rótulos e ícones. $\mathrm{O}$ autor destaca ainda a alteração na tipografia, que antes utilizava Lucida Grande e Tahoma, e foram alteradas para Helvetica (em MacOS) e Arial (em Windows). Bronwlee (2014) critica esta mudança, pois os desenvolvedores a justificam por serem fontes de sistema, porém, assim são as anteriores. Desse modo, o autor presume que esta alteração esteja voltada também para as interfaces de dispositivos móveis, visto que Helvetica e Arial são fontes mais populares em smartphones e tablets, mantendo assim a consistência do layout em diferentes suportes.

Outra característica da nova interface é alteração na apresentação de links externos. Seguindo Wilson (2014), o Facebook está realmente buscando se tornar o jornal particular das pessoas e para isso começou a utilizar uma fonte serifada para apresentar notícias e links externos (Figura 5).$$
\text { Co.Design }
$$

"With his gorgeous aerial photographs of the lvanpah Solar Electric Cenerating System--a massive expanse of solar panels in California's Mojave Desert--Jamey Stillings is hoping to expose people to a less familiar side of the green energy movement. To make it a bit more real. And ideally, to give everyone a bit more grist for the conversation.

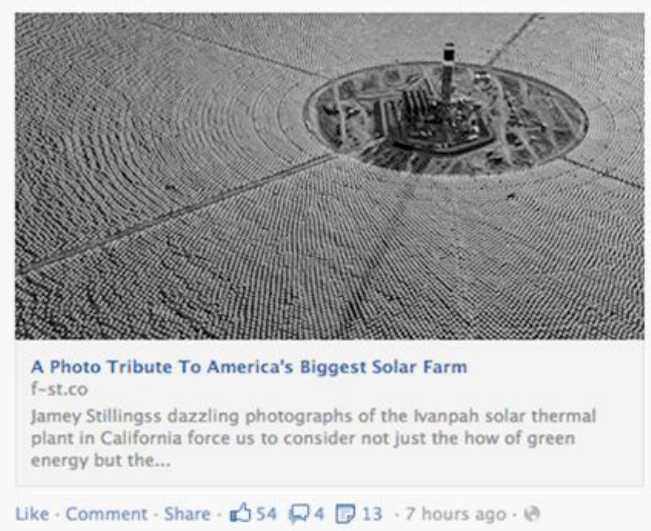

D】

With his gorgeous aerial photographs of the Ivanpah Solar Electric Gonerating Systemma massive expanso of solar panols in Callornia's Mojave Desert-Jamey Sollings is hoping to expose poople to a less familiat side of the green energy movement. To make it a bit more real. And ideally. to give everyone a bit more grist for the conversation:"

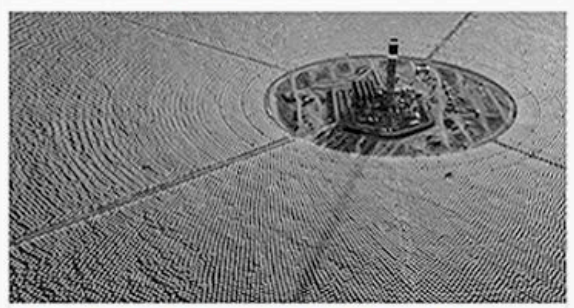

A Photo Tribute To America's Biggest Solar Farm Jame Stillingss danting photographs of the ivanput solur thermal plant in

Vkeo - Commont - Share - OSS 04013

1

Co.Design

Troo Housos: Fairy Ta'o Castlos in the Alr is a 350-page tome that collocts

Figura 5 - Uso de fonte com serifa para resgatar a ideia de jornal.

Fonte: WILSON, 2014. 
$\mathrm{Na}$ visão do autor, por mais que o Facebook queira se tornar um hub de notícias, adotar o visual de "mídias antigas" como o jornal não o levará muito longe. Brownlee (2014) também comenta este fato, afirmando que levou tanto tempo para que fosse lançada uma atualização na interface, que levará ainda mais tempo caso o Facebook esteja planejando se aproximar da experiência do papel, com informação de qualidade.

Conforme observado anteriormente, as alterações na interface da plataforma tem buscado intensificar o engajamento dos usuários por meio do destaque visual das publicações com contraste figura-fundo e uso de imagem maiores. Todavia, há também atualizações na programação da plataforma. De acordo com Brownlee (2014), novos algoritmos calculam a interação dos usuários com diferentes conteúdos e pessoas, a fim de modificar a ordem de apresentação das publicações no feed de atividades. A opção padrão de ordenamento dos posts agora segue as histórias mais importantes. Isso significa que nem sempre a publicação mais recente aparecerá no topo da linha do tempo. No lugar dela, a plataforma sugere a publicação que considerar mais relevante e atual, naquele momento, para determinado usuário.

Além dos apontamentos feitos pelos autores supracitados, outros fatores precisam ser observados, entre eles a acessibilidade. O fato de o conteúdo se tornar cada vez mais visual implica fortemente no acesso de pessoas cegas ou deficientes visuais, que representam a maior parcela dos usuários deficientes, segundo Nielsen (2000). Usuários cegos navegam a partir de leitores de tela e as imagens não podem ser lidas, a não ser que carreguem descrição textual. Atualmente o Facebook apresenta uma página ${ }^{1}$ voltada para este assunto, onde apresenta as melhorias que vem estudando e implementando para garantir uma melhor experiência para todos os tipos de usuários. Wu (2014), uma das responsáveis pelo campo de acessibilidade da plataforma, afirma que ainda há muito a ser desenvolvido e que o foco está voltado para dispositivos móveis, que por possuírem interfaces mais simples, apresentam maior grau de acessibilidade em comparação à interface desktop.

Com relação à simplificação da interface, esta é uma tendência das plataformas com grande quantidade de informação. Neste caso, conhecer o usuário é crucial (PREECE et al, 2013). A alteração do menu com rótulos para o formado apenas por ícones "pobres" é um dos erros comuns de usabilidade, apontado por Preece et al (2013). Os autores afirmam que há diferença entre reconhecer e lembrar: quando um ícone exige que o usuário lembre do que ele se trata, há um esforço cognitivo muito maior do que o ícone com fácil reconhecimento. Observa-se que o menu apresentado na interface proposta em 2013 era composto basicamente por ícones, alguns de fácil reconhecimento e outros que exigiam uma carga maior de memória. Este fato leva à retomada dos rótulos textuais no layout de 2014.

Além disso, para lidar com grandes quantidades de informação, dispositivos de reconhecimento do usuário e mapeamento dos seus padrões de navegação permitem a recomendação na forma de plataformas adaptativas. Esta característica pode ser encontrada no feed de notícias do Facebook, que sugere publicações recentes e relevantes com base na interação prévia usuário.

\footnotetext{
${ }^{1}$ https://www.facebook.com/accessibility
} 


\section{CONSIDERAÇÕES FINAIS}

Este artigo apresentou uma análise das recentes transformações na interface do Facebook, a plataforma de rede social mais utilizada no mundo. Por meio de notícias e da opinião de diferentes autores, analisou-se o layout atual que apresenta um visual mais limpo e organizado. A alteração da cor de fundo para cinza claro, o aumento das imagens e a divisão dos posts em módulos separados - cartas, destacam o conteúdo central, onde são apresentadas histórias de amigos e páginas que o usuário segue, assim como anúncios publicitários. Os campos para inserção de conteúdo continuam aparentes, sugerindo os locais e formas para o usuário pode contribuir.

Com base nas mudanças recentes da interface, é possível afirmar que estilo de interação influência fortemente o layout, principalmente em mídias onde o conteúdo é formado pelo próprio usuário, como o Facebook. A facilidade na produção e no compartilhamento de imagens propiciado pelos dispositivos móveis explicita essa necessidade, que não deve estagnar na fotografia. Grande parte das redes sociais já permite compartilhamento de conteúdo em vídeo, como o Instagram. Em pouco tempo a facilidade que se tem hoje para produzir e compartilhar fotos se estenderá para outras mídias.

Nesse sentido, cabe aos designers de interfaces compreender estas mudanças e propor estruturas que permitam que o usuário colabore além do texto. Assim como, abre-se espaço para pesquisas sobre a crescente presença dos elementos visuais na comunicação, em websites, redes sociais, e-mails e aplicativos de mensagens instantâneas.

\section{REFERÊNCIAS}

ADAMS, Paul. Why cards are the future of the web, Intercom. Disponível em <http://insideintercom.io/why-cards-are-the-future-of-the-web>. Acesso em 20 abr. 2014.

BONSIEPE, Gui. Design: do material ao digital. Florianópolis: FIESC/IEL, 1997.

BROWNLEE, Josh. Facebook Releases A Cleaner, Mobile-Friendly News Feed, FastCoDesign, 7 mar. 2014. Disponivel em <http://www.fastcodesign.com/3027377/facebook-launches-redesigned-news-feed>. Acesso em 20 abr. 2014.

CONSTINE, Josh. Facebook Launches Feeds For Photos, Music, Friends-Only, And More. TechCrunch, 7 mar. 2013.2 Disponível em: $<$ http://techcrunch.com/2013/03/07/facebook-launches-multiple-topic-based-feedsbigger-images-and-a-consistent-design-across-devices/>. Acesso em 20 abr. 2014.

COSENZA, Vicenzo. Word map of Social Networks, VINCOSBLOG, Disponível em <http://vincos.it/world-map-of-social-networks>. Acesso em 20 abr. 2014.

DUGGAN, M.; SMITH, A.. Social Media Update 2013. Disponível em: http://www.pewinternet.org/2013/12/30/social-media-update-2013/. Acesso em mar. 2014. 
FACEBOOK. A new look for news feed, Facebook Studio, 7 mar. 2013. Disponível em <https://www.facebook-studio.com/news/item/a-new-look-for-news-feed>. Acesso em 20 abr. 2014.

An Updated News Feed, Facebook for business, 12 mar. 2014. Disponível em <https://www.facebook.com/business/news/an-updated-news-feed-uk>. Acesso em 20 abr. 2014.

FOLHA. Facebook supera 1 bilhão de usuários, diz Zuckerberg. Folha de São Paulo, 4 de outubro de 2012.2 Disponível em: <http://www1.folha.uol.com.br/tec/2012/10/1163723-facebook-supera-1-bilhao-deusuarios-diz-zuckerberg.shtml>. Acesso em mar 2014.

. Nova interface do Facebook chega até abril e terá mais espaço para fotos. Folha de São Paulo - tec, 06 de março de 2014. Disponível em: <http://www1.folha.uol.com.br/tec/2014/03/1421757-com-mais-espaco-para-fotosnova-interface-do-facebook-chega-ate-abril.shtml>. Acesso em mar. 2014

GIL, Antonio Carlos. Como elaborar projetos de pesquisa. 4. ed. São Paulo: Atlas, 2008.

GLOBO, O. Queda no número de acessos ao Twitter no Brasil ultrapassa os $24 \%$. 0 Globo - Sociedade e Tecnologia, 01 de setembro de 2012. Disponível em: <http://oglobo.globo.com/sociedade/tecnologia/queda-no-numero-de-acessos-aotwitter-no-brasil-ultrapassa-os-24-5976950>. Acesso em mar. 2013.

IBOPE. Número de usuários de redes sociais ultrapassa $\mathbf{4 6}$ milhões de brasileiros. Disponível em <http://www.ibope.com.br/pt-br/noticias/Paginas/Numero-deusuarios-de-redes-sociais-ultrapassa-46-milhoes-de-brasileiros.aspx>. Acesso em 20 abr. 2014.

ISO 9241-11. Ergonomic requirements for office work with visual display terminals (VDTs) -- Part 11: Guidance on usability. 1998.

JOHNSON, Steven. Cultura da Interface - como o computador transforma nossa maneira de criar e comunicar. Rio de Janeiro: Jorge Zahar, 2001.

LINKEDIN. Press center. Disponível em: <http://press.linkedin.com/products>. Acesso em mar. 2014.

LUNDEN, Ingrid. Facebook Rolls Out Simplified News Feed That Leaves Content And Ads Alone, TechCrunch, 6 mar. 2014. Disponível em <http://techcrunch.com/2014/03/06/facebook-is-rolling-out-a-more-simplified-newsfeed-with-helveticaarial-fonts-new-cards-bolder-photos/> Acesso em 20 abr. 2014.

NIELSEN, J. Designing Web Usability - The Practice of Simplicity. USA: New Readers Publishing, 2000.

NORMAN, Donald A. Design emocional. Rio de Janeiro: Rocco, 2008.

PINTEREST. About Pinterest. Disponível em: <http://www.pinterest.com/about>. Acesso em mar. 2014.

PREECE, Jennifer, ROGERS, Ivone, SHARP, Helen. Design de Interação - além da Interação homem-computador. Porto Alegre: Bookman, 2013. 
TWITTER. About Twitter. Disponível em: <http://about.twitter.com>. Acesso em mar. 2014.

WILSON, Mark. Facebook Is Testing A News Feed That Looks More Like A Newspaper, FastCoDesign, $20 \quad$ jan. 2014.2 Disponível em <http://www.fastcodesign.com/3024667/facebook-is-testing-a-news-feed-that-looksmore-like-a-newspaper\#4>. Acesso em 20 abr. 2014.

WU, Shaomei. Developer Spotlight. Disponível em $<$ https://www.facebook.com/notes/facebook-accessibility/developer-spotlightshaomei-wu-data-scientist/710307672346294> Acesso em 20. abr. 2014. 